\title{
Qualidade da assistência em uma unidade de terapia intensiva para prevenção de lesão por pressão
}

\section{Quality of care in an intensive care unit for the prevention of pressure injuries
Calidad de asistencia en una unidad de cuidados intensivos para prevenir lesiones por presión

\begin{abstract}
Ruhama de Oliveira Rebouças ${ }^{1}$, Amelina de Brito Belchior 1, , Antonio Dean Barbosa Marques', Sarah Vieira Figueiredo", Rhanna Emanuela Fontenele Lima de Carvalho', Sherida Karanini Paz de Oliveira ${ }^{1}$
\end{abstract}

ORCID IDs

Rebouças RO (D) https://orcid.org/0000-0003-0518-5692

Belchior AB (D) https://orcid.org/0000-0002-3420-594X

Marques ADB (D) https://orcid.org/0000-0001-8969-1546

Figueiredo SV (D) https://orcid.org/0000-0003-1014-086X

Carvalho REFL (D) https://orcid.org/0000-0002-3406-9685

Oliveira SKP (D) https://orcid.org/0000-0003-3902-8046

\section{COMO CITAR}

Rebouças RO; Belchior AB; Marques ADB; Figueiredo SV; Carvalho REFL; Oliveira SKP. Qualidade da assistência em uma unidade de terapia intensiva para prevenção de lesão por pressão. ESTIMA, Braz. J. Enterostomal Ther., 2020, 18: e3420. https://doi.org/10.30886/ estima.v18.947_PT

\section{RESUMO}

Objetivo: identificar as práticas seguras para prevenção de lesão por pressão (LP), realizadas por enfermeiros em uma unidade de terapia intensiva (UTI) e classificar a qualidade da assistência. Método: estudo transversal realizado com 11 enfermeiros de uma UTI de um hospital em Fortaleza - Ceará, por meio de um questionário de prevenção de lesão por pressão para pediatria adaptado, em outubro e novembro de 2018. Utilizaram-se estatística descritiva e o Índice de Positividade (IP) da qualidade da assistência. Resultados: verificou-se uma assistência sofrível, segundo o IP, nos três domínios: medidas preventivas e detecção precoce de LP (IP: 66,6\%+24,5); medidas de alívio de pressão (IP: 41,9\%+21,6) e avaliação e notificação (IP: 65,1\%+14,5), com IP médio geral igual a 57,8\% (Desvio Padrão: $+13,8$ ), e ações de prevenção realizadas de forma inadequada. Conclusão: foi possível identificar boas práticas escassas, o que implica em uma assistência sofrível e insegura. É urgente o planejamento e a implementação de estratégias de melhorias com vistas à segurança do paciente e qualidade da assistência.

DESCRITORES: Lesão por pressão; Estomaterapia; Segurança do paciente; Cuidados de enfermagem; Qualidade da assistência à saúde.

1. Universidade Estadual do Ceará - Fortaleza (CE), Brasil.

*Autora correspondente: amelinabelchior@hotmail.com

Recebido: Set. 22, 2020 | Aceito: Dez. 06, 2020 
ABSTRACT

Objective: to identify safe practices for the prevention of pressure injury (PI), performed by nurses in an intensive care unit (ICU) and to classify the quality of care. Method: cross-sectional study conducted with 11 nurses in an ICU of a hospital in Fortaleza - Ceará, using a pressure injury prevention questionnaire adapted for pediatrics, in October and November 2018. Descriptive statistics and the Positivity Index (PIX) of the quality of care were used. Results: there was a poor assistance, according to the PIx, in the three domains: preventive measures and early detection of LP (PIx: $66.6 \%+24.5)$; pressure relief measures (PIx: $41.9 \%+21.6)$ and assessment and notification (PIx: 65.1\% + 14.5), with a general average PIx equal to 57.8\% (Standard Deviation: +13 , 8), and preventive actions carried out inappropriately. Conclusion: it was possible to identify scarce good practices, which implies poor and insecure assistance. It is urgent to plan and implement improvement strategies with a view to patient safety and quality of care.

DESCRIPTORS: Pressure injury; Stomatherapy; Patient safety; Nursing care; Quality of health care.

\section{RESUMEN}

Objetivo: La lesión por presión es un indicador de la calidad de la atención considerada un evento adverso evitable. Por tanto, es importante que las enfermeras realicen medidas preventivas. El objetivo fue identificar prácticas seguras para la prevención de lesiones por presión realizadas por enfermeros en una unidad de cuidados intensivos y clasificar la calidad de la atención. Estudio transversal realizado con 11 enfermeros de una unidad de cuidados intensivos de un hospital de Fortaleza-Ceará, utilizando un cuestionario adaptado de Prevención de Lesiones por Presión para Pediatría, en octubre y noviembre de 2018. Se utilizó estadística descriptiva y el Índice. Positividad de la calidad (IP). El estudio fue aprobado por el comité de ética con el dictamen 2.931.257. Encontró una asistencia pobre, según el IP, en los tres dominios: Medidas preventivas y Detección temprana de LP (IP: 66,6\% + 24,5); Medidas de alivio de presión (PI: 41,9\% + 21,6) y Evaluación y notificación (PI: 65,1\% + 14,5), con un PI medio general igual al 57,8\% (DE: +13, 8), y acciones preventivas realizadas de manera inadecuada. Se pudo identificar buenas prácticas escasas, lo que implica una atención deficiente e insegura. Es urgente planificar e implementar estrategias de mejora con miras a la seguridad del paciente y la calidad de la atención.

DESCRIPTORES: Lesión por presión; Estomaterapia; Seguridad del paciente; Cuidado de enfermera; Calidad de la asistencia sanitaria.

\section{INTRODUÇÃO}

Para a Organização Mundial de Saúde (OMS), qualidade significa um alto nível de excelência profissional, o uso eficiente dos recursos, o mínimo de riscos para o paciente, um alto grau de satisfação por parte do paciente e resultados finais em saúde ${ }^{1}$.

Nesse sentido, a lesão por pressão (LP) é um indicador da qualidade da assistência de enfermagem por ser considerado um evento adverso evitável. Assim, é importante que os profissionais realizem boas práticas com vistas à segurança do paciente. Medidas de prevenção podem reduzir a ocorrência desse evento adverso que está ligado intimamente com a segurança do paciente ${ }^{2}$.

A OMS definiu segurança do paciente como a diminuição ao mínimo aceitável dos riscos de danos desnecessários durante a atenção em saúde e reconhece as LP como evento adverso ${ }^{3}$. Os eventos adversos podem levar a complicações indesejáveis, o que compromete a segurança do paciente e representam um dos maiores desafios para a qualidade no setor saúde 4 . São inúmeros os danos ao paciente decorrentes das LP, tais como: dor, risco elevado de sepse, aumento do tempo de internação e da taxa de mortalidade, inevitabilidade de correções cirúrgicas e aumento dos custos hospitalares 5 .
Estudos internacionais mostram que as taxas de incidência de LP em unidade de terapia intensiva (UTI) podem variar de $8,1 \%$, a $39,3 \%{ }^{6,7}$. No Brasil, estudos realizados em UTI mostram que essas taxas variam entre $10,8 \%$ a $47 \%{ }^{8,9}$.

Nesse contexto, a Agência Nacional de Vigilância Sanitária (ANVISA) elaborou e divulgou protocolo que visa às boas práticas para prevenção de LP no serviço de saúde, prevenindo os riscos e eventos adversos ${ }^{10}$. As boas práticas são o conjunto das técnicas, processos, procedimentos e atividades identificados, utilizados, comprovados e reconhecidos por diversas organizações em determinada área do saber, como sendo os melhores quanto ao mérito, eficácia e sucesso alcançados pela sua aplicação na realização de uma tarefa, nesse caso a prevenção de $\mathrm{LP}^{11}$.

Desse modo, o enfermeiro que está diretamente ligado ao cuidado também é responsável pela segurança do paciente e cuidados com a pele do paciente, promovendo saúde e prevenindo complicações, erros e eventos adversos ao realizar as ações de boas práticas ${ }^{10}$. Considerando as LP como um indicador de qualidade de assistência de enfermagem, reduzir seu número é papel primordial da equipe de enfermagem por meio de estratégias e ações contínuas de prevenção ${ }^{12}$.

Dada a importância e o impacto da LP e por ser considerada um evento adverso passível de prevenção por 
meio de boas práticas nos serviços de saúde, surgiu o seguinte questionamento: quais ações de prevenção de LP estão sendo realizadas em UTI?

Acredita-se que o presente estudo poderá subsidiar informações importantes de avaliação e oportunidades de melhorias aos enfermeiros, gestores e equipe de saúde, com vistas à redução de eventos adversos por meio de estratégias efetivas de prevenção e práticas seguras relacionadas à LP.

Ante o exposto, objetivou-se identificar as práticas seguras para prevenção da LP realizadas por enfermeiros em UTI e classificar a qualidade da assistência.

\section{MÉTODOS}

Estudo transversal de abordagem quantitativa, realizado em uma UTI de um hospital público de referência na área de cardiologia e pneumologia em Fortaleza - Ceará, nos meses de outubro e novembro de 2018.

A população foi composta por 70 enfermeiros que atuavam na UTI. Ressalta-se que 18 enfermeiros estavam de férias ou licença, 37 não devolveram os questionários até o prazo combinado, mesmo esse tempo sendo estendido por mais 5 dias, e 4 recusaram participar da pesquisa.

A amostra foi composta por 11 enfermeiros. O recrutamento para compor a amostra foi realizado a partir dos critérios de inclusão: ser enfermeiro assistencial há, pelo menos, seis meses e ter disponibilidade para o preenchimento do questionário. Foram excluídos os enfermeiros que estavam de férias, licença ou afastados de suas atividades no período da coleta de dados.

Utilizou-se um formulário com dados sociodemográficos e profissionais e o questionário de prevenção de lesão por pressão para pediatria (PLPP) ${ }^{13}$ adaptado. Esse instrumento foi construído segundo o "Protocolo para Prevenção de Úlcera por Pressão" do Ministério da Saúde ${ }^{14}$ para avaliação de ações de prevenção na pediatria, porém apresenta cuidados que não diferem entre o público infantil e adulto. Por isso optou-se pela sua utilização, uma vez que apresentou boa validade (Coeficiente de Correlação Intraclasse - ICC= 0,983) e confiabilidade (alfa de Cronbach $=0,938)^{13}$.

Esse questionário possui 23 itens divididos em 3 domínios, quais sejam: medidas preventivas e detecção precoce de LP (9 itens); medidas de alívio de pressão (8 itens) e avaliação e notificação (6 itens), distribuídos em escala Likert de 1 a $5^{13}$.
Os dados foram tabulados e organizados em uma planilha do aplicativo Microsoft Excel $^{\circledR}$, analisados por meio de estatística descritiva simples e apresentados em tabelas, conforme os domínios da escala utilizada.

Para a avaliação da qualidade assistencial, Haddad $(2004)^{15}$ propôs um instrumento que avalia a estrutura, o processo e o resultado, disponibilizando um roteiro de avaliação da realidade do serviço oferecido ao paciente em relação à assistência de enfermagem de forma integral. Essa avaliação evidencia a realidade do serviço e proporciona subsídios para planejamentos de intervenções com intuito de melhorar e aperfeiçoar a qualidade da assistência.

Ademais, identificadas as intervenções realizadas pelos profissionais, a qualidade da assistência e as boas práticas foram analisadas por meio do Índice de Positividade (IP) e da Qualidade da Assistência (QA) ${ }^{15}$.

Para classificar a QA, os dados foram analisados de acordo com o IP, que se refere ao percentual de respostas positivas, e classificadas em: assistência desejável (100\% de positividade); assistência adequada ( 90 a 99\% de positividade); assistência segura ( 80 a $89 \%$ de positividade); assistência limítrofe (71 a 79\% de positividade); e assistência sofrível (inferior a $70 \%$ de positividade) ${ }^{15}$. Considerou-se resposta positiva na escala de Likert "sempre efetua essa conduta em seu cotidiano de trabalho", uma vez que as práticas seguras devem sempre estar presentes no cuidado de saúde, contribuindo para segurança do paciente.

O estudo obedeceu à Resolução no 466/2012 do Conselho Nacional de Saúde ${ }^{16}$ e foi aprovado pelo Comitê de Ética em Pesquisa com Seres Humanos sob o parecer no 2.931.257 e CAAE no 97887618.2.0000.5039.

\section{RESULTADOS}

A Tabela 1 mostra os dados referentes ao perfil sociodemográfico e profissional dos enfermeiros respondentes do questionário de ações de prevenção de LP.

A maioria dos participantes foi composta por mulheres $(54,5 \%)$, porém observa-se um quantitativo equilibrado entre homens e mulheres, diferente do que se espera na enfermagem. A média de idade foi de $33( \pm 5,1)$ anos, exibindo um perfil de profissionais jovens; $6,4( \pm 5,2)$ anos para formação, e 3,5 $( \pm 3,9)$ anos sobre o tempo de atuação no serviço, despontando certa experiência dos profissionais. Dos participantes, $2(18,1 \%)$ eram enfermeiros estomaterapeutas. 
Tabela 1. Características sociodemográficas dos enfermeiros. Fortaleza (CE), Brasil - 2018.

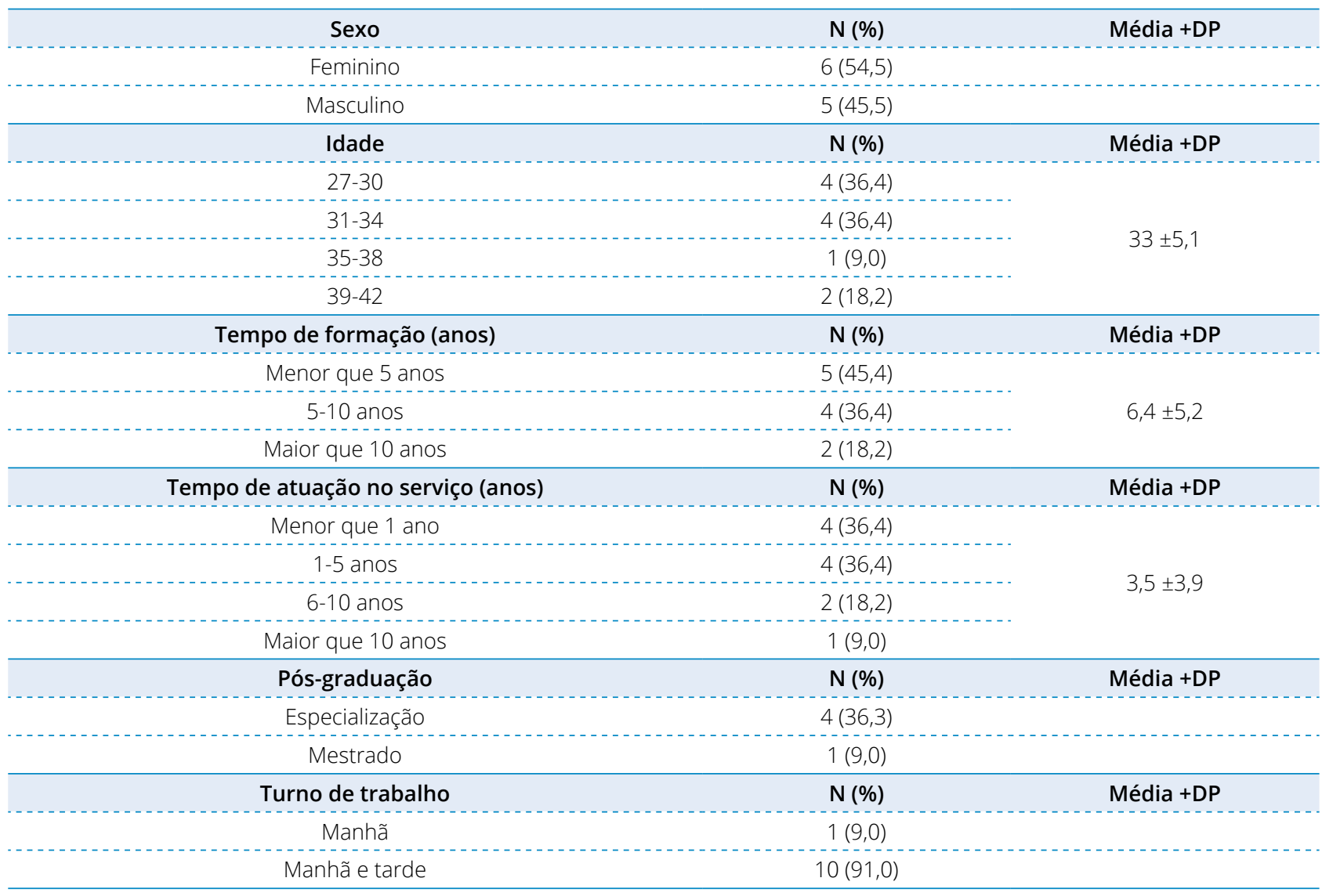

DP = Desvio Padrão. *Dois são enfermeiros especialistas em estomaterapia.

A Tabela 2 exibe os dados referentes à frequência de ações de prevenção realizadas pelos enfermeiros participantes do estudo e ao IP da QA.

Sobre o domínio 1 , medidas preventivas relacionadas às boas práticas e detecção precoce de LP, encontrou-se que $10(90,9 \%)$ enfermeiros sempre realizam a limpeza da pele e hidratação da pele ressecada; 9 (81,8\%) sempre realizam a inspeção da pele dos pacientes na admissão; e 4 (36,3\%) evitam massagear áreas de proeminência óssea ou hiperemiada.

O domínio 2 apresenta os dados das medidas de alívio da pressão e mostra que apenas 5 (45\%) enfermeiros sempre realizavam mudança de decúbito a cada 2 horas como também providenciavam superfícies de apoio para calcâneo, revelando um baixo indicador de medidas importante de prevenção de LP.

As ações de oferecer apoio sob os pés dos pacientes e providenciar superfície de redistribuição de pressão são apontadas por 6 (54\%) enfermeiros, evidenciando pouca preocupação em evitar o atrito com a cama e redistribuição e alívio da pressão, com o intuito de promover circulação eficaz nos membros inferiores dos pacientes.
Sobre a comunicação de medidas de alívio da pressão, somente 1 enfermeiro afirmou utilizar quadro de avisos e 3 (27\%) informaram que essa medida não se aplica, talvez pela inexistência desse instrumento no local estudado.

Em relação ao domínio 3, avaliação e notificação das LP, a avaliação do risco de LP na admissão e reavaliação diária, utilizando a Escala de Braden, obteve adesão de $8(72,7 \%)$ e $7(63,6 \%)$ enfermeiros, respectivamente, apresentando bom IP.

Quando questionados sobre avaliação de sinais clínicos de desnutrição e notificação ao nutricionista sobre o risco nutricional, somente 6 (54,5\%) enfermeiros fazem avaliação do estado nutricional do paciente, identificando as necessidades nutricionais.

O registro no prontuário sobre as alterações detectadas na pele e intervenções foi indicado por 10 (90,9\%), demonstrando que existe comunicação entre a equipe por meio do prontuário. Também sobre comunicação, 6 (54,5\%) enfermeiros assinalaram notificar à Gerência de Riscos ou Núcleo de Segurança do Paciente sobre a deteç̧ão de LP. 
Tabela 2. Índice de Positividade das práticas de prevenção de lesão por pressão em uma unidade de terapia intensiva. Fortaleza (CE), Brasil - 2018.

\begin{tabular}{|c|c|c|c|c|c|c|c|}
\hline Itens & Nunca & $\begin{array}{l}\text { Quase } \\
\text { nunca }\end{array}$ & $\begin{array}{c}\text { Às } \\
\text { vezes }\end{array}$ & $\begin{array}{l}\text { Quase } \\
\text { sempre }\end{array}$ & Sempre & $\begin{array}{l}\text { Não se } \\
\text { aplica }\end{array}$ & \multirow{2}{*}{ IP } \\
\hline $\begin{array}{l}\text { Medidas preventivas e detecção } \\
\text { precoce de LP }\end{array}$ & n (\%) & n (\%) & n (\%) & n (\%) & n (\%) & n (\%) & \\
\hline 1-Inspeção da pele na admissão & - & - & - & $2(18,2)$ & $9(81,8)$ & - & 81,8 \\
\hline 2-Inspeção da pele diária & $1(9,1)$ & - & - & $3(27,3)$ & $6(54,5)$ & $1(9,1)$ & 54,5 \\
\hline 3-Limpeza da pele & - & & - & $1(9,1)$ & $10(90,9)$ & - & 90,9 \\
\hline 4-Orientação quanto à limpeza da pele & $1(9,1)$ & - & $3(27,3)$ & - & $6(54,5)$ & $1(9,1)$ & 54,5 \\
\hline 5-Hidratação da pele ressecada & - & - & - & $1(9,1)$ & $10(90,9)$ & - & 90,9 \\
\hline $\begin{array}{l}\text { 6-Evita massagear áreas de proeminência } \\
\text { óssea ou hiperemiada }\end{array}$ & $1(9,1)$ & $1(9,1)$ & $1(9,1)$ & $3(27,3)$ & $4(36,4)$ & $1(9,1)$ & 36,3 \\
\hline $\begin{array}{l}\text { 7-Protege a pele da exposição } \\
\text { à umidade excessiva }\end{array}$ & - & - & $1(9,1)$ & $1(9,1)$ & $8(72,7)$ & $1(9,1)$ & 72,7 \\
\hline $\begin{array}{l}\text { 8-Evita posicionar o paciente } \\
\text { diretamente sobre cateteres }\end{array}$ & - & - & - & $1(9,1)$ & $10(90,9)$ & - & 90,9 \\
\hline 9- Utiliza coberturas para proteger a pele & - & $1(9,1)$ & $4(36,4)$ & $2(18,2)$ & $3(27,3)$ & $1(9,1)$ & 27,2 \\
\hline Medidas de alívio da pressão & n (\%) & n (\%) & $\mathrm{n}(\%)$ & $\mathrm{n}(\%)$ & $\mathrm{n}(\%)$ & $\mathrm{n}(\%)$ & IP \\
\hline 10-Mudança de decúbito a cada 2 horas & - & - & $2(18,2)$ & $3(27,3)$ & $5(45,4)$ & $1(9,1)$ & 45,4 \\
\hline $\begin{array}{l}\text { 11-Realiza o reposicionamento do paciente com } \\
\text { ventilação não-invasiva }\end{array}$ & - & - & $1(9,1)$ & $2(18,2)$ & $7(63,6)$ & $1(9,1)$ & 63,6 \\
\hline 12-Oferece apoio sob os pés do paciente & - & - & $1(9,1)$ & $3(27,3)$ & $6(54,5)$ & $1(9,1)$ & 54,5 \\
\hline $\begin{array}{l}\text { 13-Providencia superfície de } \\
\text { redistribuição de pressão }\end{array}$ & - & - & - & $5(45,5)$ & $6(54,5)$ & - & 54,5 \\
\hline 14-Providencia superfícies de apoio para calcâneo & - & $2(18,2)$ & $1(9,1)$ & $2(18,2)$ & $5(45,4)$ & $1(9,1)$ & 45,4 \\
\hline $\begin{array}{l}\text { 15-Providencia assento de } \\
\text { redistribuição de pressão }\end{array}$ & $1(9,1)$ & $2(18,2)$ & $1(9,1)$ & - & $1(9,1)$ & $6(54,5)$ & 9,0 \\
\hline $\begin{array}{l}\text { 16-Usa forro ou dispositivo de } \\
\text { elevação para mover paciente }\end{array}$ & - & $1(9,1)$ & $1(9,1)$ & $1(9,1)$ & $6(54,5)$ & $2(18,2)$ & 54,5 \\
\hline 17- Utiliza quadro de avisos & $1(9,1)$ & $2(18,2)$ & $2(18,2)$ & $2(18,2)$ & $1(9,1)$ & $3(27,2)$ & 9,0 \\
\hline Avaliação e Notificação & $n(\%)$ & $\mathrm{n}(\%)$ & $\mathrm{n}(\%)$ & $\mathrm{n}(\%)$ & $\mathrm{n}(\%)$ & $\mathrm{n}(\%)$ & IP \\
\hline $\begin{array}{l}\text { 18-Avalia o risco de LP na admissão } \\
\text { utilizando Escala de Braden }\end{array}$ & - & $1(9,1)$ & $2(18,2)$ & - & $8(72,7)$ & - & 72,7 \\
\hline $\begin{array}{l}\text { 19-Reavalia diariamente o risco de LP } \\
\text { utilizando a Escala de Braden }\end{array}$ & - & - & $1(9,1)$ & $2(18,2)$ & $7(63,6)$ & $1(9,1)$ & 63,6 \\
\hline 20-Avalia sinais clínicos de desnutrição & - & - & $1(9,1)$ & $4(36,4)$ & $6(54,5)$ & - & 54,5 \\
\hline 21-Notifica o nutricionista o risco nutricional & - & $1(9,1)$ & $1(9,1)$ & $3(27,3)$ & $6(54,5)$ & - & 54,5 \\
\hline $\begin{array}{l}\text { 22-Registra no prontuário as alterações } \\
\text { detectadas na pele e intervenções }\end{array}$ & - & $1(9,1)$ & - & - & $10(90,9)$ & - & 90,9 \\
\hline $\begin{array}{l}\text { 23-Notifica a Gerência de Riscos ou } \\
\text { Núcleo de Segurança do Paciente. }\end{array}$ & $1(9,1)$ & $1(9,1)$ & $2(18,2)$ & $1(9,1)$ & $6(54,5)$ & - & 54,5 \\
\hline
\end{tabular}

LP = Lesão por Pressão; IP = Índice de Positividade. 
Ressalta-se que no instrumento constava um espaço para o enfermeiro escrever outras ações realizadas para a promoção da segurança dos pacientes quanto à prevenção de LP, mas nenhum profissional citou qualquer outra ação realizada.

Em relação ao IP, dos 9 itens do domínio 1, 3 itens obtiveram IP maior que $90 \%$ (assistência adequada) e 1 item obteve IP entre 80 e $89 \%$ (assistência segura). O IP médio foi de $66,6 \%( \pm 24,5)$, variando de 90,9 a $27,2 \%$, sendo, portanto, classificado como sofrível.

Todos os itens do domínio 2 obtiveram IP menor que 63,6\%, com média de 41,9\% $( \pm 21,6)$, considerado assistência sofrível, sendo as ações Providencia assento de redistribuição de pressão e Utiliza quadro de avisos com pior IP (9,0\%).

O IP do domínio 3 do instrumento PLPP, Avaliaçãoe Notificação, também atingiu classificação considerada como assistência sofrível, $65,1 \%( \pm 14,5)$, apesar de 2 itens, 22 e 18, terem alcançado IP de 90,9\% (assistência adequada) e $72,7 \%$ (assistência limítrofe), respectivamente.

Considerando a escala completa, pode-se dizer que os cuidados relacionados às boas práticas para prevenção de LP podem ser classificados como sofríveis, já que obtiveram IP médio geral igual a 57,8\% $( \pm 13,8)$. Indicador preocupante, pois aponta para um baixo percentual de realização de medidas importantes que garantem a avaliação de riscos e a implementação de ações com vistas à redução de erros e danos aos pacientes. Ademais, vislumbra-se a necessidade de mais investimentos em materiais e capacitações para a equipe de saúde.

\section{DISCUSSÃO}

\section{Domínio 1: medidas preventivas e detecção precoce de lesão por pressão}

Encontrou-se que $81,8 \%$ dos enfermeiros realizavam Inspeção na pele do paciente na admissão. Assim, classifica-se como assistência segura, tendo em vista que a maioria dos enfermeiros realizava essa avaliação. Em contrapartida, apenas 54,5\% dão continuidade a essa inspeção diariamente, sendo classificada como assistência sofrível. Algumas das ações mais realizadas pelos enfermeiros encontradas no presente estudo foram Limpeza da pele e Hidratação da pele ressecada.

Dado corroborado por um estudo bibliográfico, quanto às medidas preventivas foram mencionadas ações de prevenção que contemplam a inspeção, higienização e hidratação ${ }^{17}$. Em contrapartida, em outro estudo de revisão integrativa, a limpeza de pele foi pouco citada e reconhecida como cuidado para prevenção de LP, embora a enfermagem esteja atuando diariamente na higienização da pele dos pacientes ${ }^{18}$. O mesmo estudo indicou que aproximadamente $60 \%$ dos profissionais de enfermagem realizam hidratação da pele dos pacientes. Esse cuidado constitui importante medida preventiva a ser feita pela equipe de enfermagem. Contudo, ao comparar dois grupos de pacientes, o grupo sem LP recebeu mais vezes esse cuidado quando comparado ao grupo com $\mathrm{LP}^{18}$.

A maioria dos enfermeiros neste estudo evita posicionar o paciente diretamente sobre cateteres para impedir o desenvolvimento de lesão por pressão relacionada a dispositivos médicos (LPRDM). A simples colocação de um dispositivo médico já é o ponto de partida para formação de uma LPRDM. Observa-se que os pacientes críticos são mais suscetíveis a desenvolver lesões pelo uso e o tempo de permanência do dispositivo, devido à má perfusão dos tecidos causada pelo uso de drogas vasoativas ${ }^{19}$.

O IP no domínio 1 evidenciou uma assistência sofrível e a necessidade de investimentos na capacitação e incentivo dos profissionais para realização de medidas de prevenção, por ser menos dispendioso e de menor impacto para o paciente.

\section{Domínio 2: medidas de alívio da pressão}

Nas ações do domínio 2, os dados obtidos evidenciaram baixa adesão dos profissionais a todos os itens, revelando um baixo indicador de uma medida importante para alívio da pressão sobre proeminência óssea e uma circulação adequada do tecido, assim como para evitar o atrito com a cama.

$\mathrm{O}$ item Realiza o reposicionamento do paciente com ventilação não invasiva obteve maior adesão $(63,6 \%)$ dos profissionais, e os itens Providencia assento de redistribuição de pressão e Utiliza quadros de aviso, menor adesão (9\%).

Estudos apontam que a medida mais adotada para prevenção de LP em pacientes de UTI é a mudança de decúbito $^{20,21}$.Portanto, esses dados reforçam a necessidade de capacitação profissional contínua e melhor dimensionamento de profissionais para atender as necessidades das unidades e atualizações periódicas.

Os locais com maior predisposição para o desenvolvimento de LP é região sacra e os calcâneos, localizações anatômicas que podem ser protegidas. Existem fatores não modificáveis 
e que são de riscos significativos, mas a grande maioria deles pode ser evitada caso a equipe de enfermagem atue de forma qualificada ${ }^{21,22}$.

O domínio 2 obteve o menor IP (41,9\%), classificado como assistência sofrível. Indicando um baixo índice para medidas que são essenciais para a prevenção de LP, expondo a real necessidade da instituição de investir em educação continuada e em materiais para realização efetiva de medidas de alívio da pressão.

\section{Domínio 3: avaliação e notificação}

Nesse domínio, apenas 1 item apresentou assistência adequada, Registra no prontuário as alteraçôes detectadas na pele e intervenções (90,9\%), e a média de IP desse domínio revelou assistência sofrível, apesar dos registros e notificações serem significativos e essenciais para os setores assistenciais, cabendo evidenciar que as medidas preventivas de LP são parte integrante do Programa de Segurança do Paciente ${ }^{23}$.

A Escala de Braden é um instrumento útil, de fácil manuseio, sem custo para a instituição e usado como um indicador de saúde na segurança do paciente, que auxilia o enfermeiro para a realização de uma avaliação global do risco de desenvolvimento de LP no paciente hospitalizado ${ }^{12}$.

Os dados obtidos no presente estudo evidenciaram que os enfermeiros necessitam avaliar e reavaliar mais seus pacientes a fim de identificar os riscos existentes por meios de escalas para o desenvolvimento de LP precocemente e, assim, implementarem cuidados individualizados em qualquer nível de atenção à saúde.

Pesquisa realizada em um município ao Norte do Rio Grande do Sul revelou que no julgamento dos enfermeiros a escala de Braden é considerada um instrumento importante e útil na prevenção da LP. Contudo, a falta de tempo e a carga elevada de atividades favorece sua aplicação, muitas vezes, apenas para cumprir protocolo da instituição, sem uma avaliação real do paciente ${ }^{12}$.

O presente estudo verificou que $72 \%$ dos enfermeiros utilizam a escala de Braden na admissão do paciente e 63\% a utilizam diariamente na reavaliação. Esses dados ainda são considerados como uma assistência limítrofe ou sofrível, evidenciando a necessidade de encontrar estratégias de melhorias para as boas práticas no cuidado de saúde e, em especial, dessa medida simples.

A utilização da Escala de Braden para avaliar o risco do paciente desenvolver uma LP demonstrou que todos os pacientes internados na UTI apresentavam algum risco, seja baixo, moderado ou alto ${ }^{24}$. Nesse sentido, a aplicação da Escala de Braden pelo enfermeiro direciona as intervenções de enfermagem na prevenção de $\mathrm{LP}^{17}$. Por isso a importância da avaliação do risco pelo enfermeiro na UTI com vistas ao planejamento dessas ações.

Percebe-se ainda dificuldades na análise das subescalas da Escala de Braden, sendo imperativa a otimização do uso da escala bem como da interpretação correta da escala por parte dos profissionais e o incentivo para que toda a equipe realize as medidas preventivas baseadas nos riscos que a escala revela sobre determinado paciente ${ }^{25}$.

Assim, são necessárias capacitações periódicas da equipe para fornecer subsídios para implementação de estratégias que visem à diminuição de LP nessas unidades ${ }^{24}$.

Os registros e as notificações configuram-se como subsídios significativos e efetivos para uma gestão de cuidados e gestão de riscos eficazes. Portanto, há que se investir e estimular os profissionais a registrarem e notificarem os eventos adversos ${ }^{23}$.

Se não houver notificação efetiva de tais eventos adversos, não há como obter dados epidemiológicos reais nem avaliar os aspectos envolvidos. $\mathrm{Na}$ ausência de registros, há debilidade nos estudos sobre a temática e a desclassificação da assistência prestada. Reforça-se a importância da notificação como oportunidade de melhoria de um dano evitável durante os cuidados de saúde, o que merece ser revisto e reforçado entre esses profissionais. A equipe deve atuar de forma mais integrada e interdisciplinar com vistas a uma melhor assistência de saúde ao paciente.

Considerando a escala completa, pode-se dizer que os cuidados relacionados à prevenção de LP foram classificados como assistência sofrível, um indicador preocupante, pois aponta para um baixo percentual de realização de medidas simples e importantes que garantem a avaliação de riscos e a implementação de ações com vistas à redução de erros e danos aos pacientes.

Ter uma assistência sofrível significa que os processos de Gestão de Risco referentes às tecnologias em saúde, englobando ações e serviços do Programa Nacional de Segurança do Paciente no âmbito hospitalar, encontram-se com indicadores aquém do esperado para uma assistência segura.

Assim, para uma prática assistencial segura, o Ministério da Saúde orienta o uso de protocolos, planos locais de segurança do paciente dos estabelecimentos de saúde, criação dos núcleos de segurança do paciente e sistema de notificação de incidentes ${ }^{2}$. 
Dentre as estratégias de melhoria na implementação de melhores práticas, recomenda-se fornecer educação em prevenção e tratamento de LP, em nível organizacional, como parte de um plano de melhoria da QA para reduzir a incidência de $\mathrm{LP}^{26}$. Mediante o exposto, a prática fundamentada em evidências científicas deve iniciar-se durante a formação do enfermeiro, sendo fator primordial para viabilização e implementação de cuidados na prevenção e manejo da $\mathrm{LP}^{27}$.

Percebe-se que ainda há muito a ser feito dentro da perspectiva da qualidade para melhorar a assistência prestada, com foco nos processos que garantam a segurança dos pacientes ${ }^{1}$.

Como limitações, tem-se o uso de instrumentos validados específicos para população adulta e coleta de dados com número reduzido de enfermeiros.

\section{CONCLUSÃO}

Foi possível identificar que as boas práticas relacionadas à prevenção de LP não são efetuadas ou apresentam déficits na sua realização pelos enfermeiros. A utilização de um questionário válido e confiável possibilitou a mensuração da frequência de realização das boas práticas bem como o IP da QA relacionado à realização de boas práticas para prevenção de LP.

Verificou-se que, de modo geral, todas as medidas preventivas precisam de melhorias ou mesmo de implementação, já que a assistência em relação à prevenção de LP foi classificada como sofrível. Contudo, destaca-se uma assistência adequada nos quesitos: realização da limpeza da pele do paciente, hidratação da pele ressecada, evitar posicionar o paciente diretamente sobre cateteres e registro no prontuário sobre alterações detectadas na pele e intervenções. A assistência segura foi relacionada à inspeção da pele na admissão do paciente e a assistência limítrofe nos itens sobre proteção da pele da exposição à umidade excessiva e avaliação do risco de LP na admissão utilizando a Escala de Braden.

Sugerem-se investimentos em educação continuada e permanente por meio de estratégias que vão além das informações técnicas, como comunicação, inteligência emocional, trabalho em equipe, liderança, tomada de decisões e percepção da situação com vistas à sensibilização e engajamento dos profissionais para oferecer qualidade da assistência prestada.

\section{CONTRIBUIÇÃO DOS AUTORES}

Conceitualização: Rebouças RO, Carvalho REFL e Oliveira SKP; Metodologia: Rebouças RO, Marques ADB, Figueiredo SV, Carvalho REFL e Oliveira SKP; Investigação: Rebouças RO, Carvalho REFL e Oliveira SKP; Redação - Primeira versão: Rebouças RO, Belchior AB, Marques ADB, Figueiredo SV, Carvalho REFL e Oliveira SKP; Redação - Revisão \& Edição: Belchior AB e Oliveira SKP. Supervisão: Oliveira SKP.

\section{REFERÊNCIAS}

1. Albuquerque CIRA, Madeira GS, Capanema IVL, Ferreira JS. Qualidade em Saúde: Evolução e Desafios no Contexto Brasileiro. DNVGL 2017;1-11. Disponível em: https:// brandcentral.dnvgl.com/original/gallery/10651/files/original /Offb8c99f79846d2a359edd041d0a482.pdf

2. Ministério da Saúde (BR). Documento de referência para o Programa Nacional de Segurança do Paciente. Brasília (DF): Agência Nacional de Vigilância Sanitária; 2014.

3. Organização Mundial da Saúde. Guia Curricular de Segurança do Paciente da Organização Mundial da Saúde: Edição Multiprofissional. Rio de Janeiro (RJ); 2016.

4. Silva-Batalha EMS, Melleiro MM. Cultura de segurança do paciente em um hospital de ensino: diferenças de percepção existentes nos diferentes cenários desta instituição. Texto contexto - enferm 2015;24(2):432-41. https://doi.org/10.1590/0104-07072015000192014
5. Instituto Brasileiro para Segurança do Paciente. 5 erros comuns que ocorrem na assistência à saúde. 2017. [citado em 21 ago 2020]. Disponível em: https://www. segurancadopaciente.com.br/seguranca-e-gestao/5-erroscomuns-que-ocorrem-na-assistencia-a-saude/

6. González-Méndez MI, Lima-Serrano M, Martín-Castaño C, Alonso-Araujo I, Lima-Rodríguez JS. Incidence and risk factors associated with the development of pressure ulcers in an intensive care unit. J Clin Nurs 2018;27(5-6):1028-37. https://doi.org/10.1111/jocn.14091

7. Tayyib N, Coyer F, Lewis P. Saudi Arabian adult intensive care unit pressure ulcer incidence and risk factors: a prospective cohort study. Int Wound J 2016;13(5):912-9. https://doi. org/10.1111/iwj.12406

8. Silva SAM, Pires PS, Macedo MP, Oliveira LS, Batista JET, Amaral JM. Lesão por pressão: incidência em unidades 
críticas de um hospital regional. ESTIMA, Braz J Enterostomal Ther 2018;16:e4318. https://doi.org/10.30886/estima. V16.655_PT

9. Ali YCMM, Souza TMP, Garcia PC, Nogueira PC. Incidência de lesão por pressão e tempo de assistência de enfermagem em terapia intensiva. ESTIMA, Braz J Enterostomal Ther 2020;18:e1120. https://www.revistaestima.com.br/index. php/estima/article/view/849/pdf_1

10. Ministério da Saúde (BR). Nota técnica GVIMS/GGTES n03/2017: Práticas seguras para prevenção de Lesão por Pressão em serviços de saúde. Brasília (DF): Agência Nacional de Vigilância Sanitária; 2017.

11. Porto CC. Arte Clínica. Jornal do Clínico, 2002; ano X (57):11.

12. Debon R, Fortes VLF, Rós ACR, Scaratti M. A Visão de Enfermeiros Quanto a Aplicação da Escala de Braden no Paciente Idoso. J res fundam care online 2018;10(3): 81723. https://doi.org/10.9789/2175-5361.2018.v10i3.817-823

13. Ferreira MKM. Construção e validação do instrumento prevenção de lesão por pressão na pediatria [dissertação]. Fortaleza (CE): Mestrado em Enfermagem, Universidade Federal do Ceará; 2018.

14. Ministério da Saúde (BR), Anexo 2: Protocolo para prevenção de úlcera por pressão. Brasília (DF): Ministério da Saúde; 2013.

15. Haddad MCL. Qualidade da assistência de enfermagem o processo de avaliação em hospital universitário público [tese]. Ribeirão Preto (SP): Doutorado em Enfermagem, Universidade de São Paulo; 2004.

16. Resolução $n^{\circ} 466$ de 12 de dezembro de 2012 (BR). Dispõe sobre diretrizes e normas regulamentadoras de pesquisas envolvendo seres humanos. Diário Oficial da União [periódico na internet], Brasília (DF). 13 jun 2013. Disponível em: $\quad$ https://conselho.saude.gov.br/resolucoes/2012/ Reso466.pdf

17. Feitosa DVS, Silva NSO, Pereira FNM, Almeida TF, Estevam AS. Atuação do enfermeiro na prevenção de lesão por pressão: uma revisão integrativa da literatura. REAS 2020;43:e2553. Disponível em: https://acervomais.com.br/index.php/saude/ article/view/2553

18. Menezes LCG, Fernandes MMJ, Guedes MVC, Oliveira RM, Leitão IMTA, Moura DJM. Cuidados Clínicos e Gerenciais de Enfermagem na Prevenção de Úlcera por Pressão. ESTIMA, Braz J Enterostomal Ther 2017;15(2):107-14. https://doi. org/10.5327/Z1806-3144201700020007
19. Cavalcanti EO, Kamada I. Lesão por pressão relacionada a dispositivo médico em adultos: revisão integrativa. Texto Contexto Enferm 2020;29:e20180371. https://doi. org/10.1590/1980-265x-tce-2018-0371

20. Gonçalves KPO, Sabino KN, Azevedo RVM, Canhestro MR. Avaliação dos cuidados de manutenção de cateteres venosos periféricos por meio de indicadores. REME - Rev Min Enferm 2019;23:e-1251. https://doi.org/10.5935/14152762.20190099

21. França JRG, Sousa BVN, Jesus VS. Cuidados de Enfermagem na Prevenção de Lesões por Pressão em Unidades de Terapia Intensiva: uma Revisão Sistemática. Revista Brasileira de Saúde Funcional 2016;1(11):16-31. Disponível em: http:// www.seer-adventista.com.br/ojs/index.php/RBSF/article/ download/709/619/0

22. Constantin AG, Moreira APP, Oliveira JLC, Hofstätter LM, Fernandes LM. Incidência de lesão por pressão em unidade de terapia intensiva para adultos. ESTIMA, Braz J Enterostomal Ther 2018;16:e1118. https://doi. org/10.30886/estima.v16.454_PT

23. Nascimento DC, Ferreira GAS, Silva JML, Pioli MT, Decio MRM. Registro de lesão por pressão: o que é abordado? Revista HUPE 2016;15(4):343-8. https://doi.org/10.12957/ rhupe.2016.31614

24. Santos $L R C L$, Lino AIA. Riscos de lesão por pressão: aplicação da Escala de Braden em terapia intensiva. ESTIMA, Braz J Enterostomal Ther 2018;16:e0818. https://doi. org/10.30886/estima.v16.443_PT

25. Soares CF, Heidemann ITSB. Promoção da saúde e prevenção da lesão por pressão: expectativas do enfermeiro da atenção primária. Texto contexto - enferm 2018;27(2):e1630016. http://doi.org/10.1590/0104-070720180001630016

26. European Pressure Ulcer Advisory Panel, National Pressure Injury Advisory Panel and Pan Pacific Pressure Injury Alliance (EPUAP/NPIAP/PPPIA). Prevenção e tratamento de úlceras/ lesões por pressão: guia de consulta rápida (edição em português brasileiro). EmilyHaesler(Ed.): 2019. Disponível em: https://www.epuap.org/wp-content/uploads/2020/11/ qrg-2020-brazilian-portuguese.pdf

27. Bernardes RM, Caliri MHL. Construção e validação de um website sobre lesão por pressão. Acta paul enferm 2020;33:eAPE20190130. https://doi.org/10.37689/actaape/2020ao01305 